BULGARIAN ACADEMY OF SCIENCES

CYBERNETICS AND INFORMATION TECHNOLOGIES • Volume 15, No 3

Sofia $\bullet 2015$

Print ISSN: 1311-9702; Online ISSN: 1314-4081

DOI: $10.1515 /$ cait-2015-0041

\title{
Improving QoS Routing in Hybrid Wireless Mesh Networks, Using Cross-Layer Interaction and MAC Scheduling
}

\author{
Anita C. S. ${ }^{1}$, Suresh R. M. ${ }^{2}$ \\ ${ }^{1}$ Department of Computer Science and Engineering, R.M.D. Engineering College, Chennai, Tamil \\ Nadu, India \\ ${ }^{2}$ Sri Muthukumaran Institute of Technology, Mangadu, Chennai, Tamil Nadu, India \\ Emails: anitacs28377@gmail.com_rmsuresh@hotmail.com
}

Abstract: Delivering Quality of Services (QoS) for cooperative Wireless Mesh Networks $(W M N)$ is an important issue when enabling heterogeneous wireless technologies on the client side. A standard approach for satisfying the QoS requirements of different wireless clients appears to be a complex task due to the capacity variations. In addition to the routing layer, the information at the lower layers, such as Physical and Medium Access Control (MAC) layers must be considered for providing the QoS guarantee. The strength of a signal received from heterogeneous clients at the physical layer and the coupled network capacity at the MAC layer plays an important role in the efficient path assignment and time scheduling management for routing. Therefore, this paper proposes an Adaptive Multipath-Dynamic Source Routing (AM-DSR) protocol. It supports Cross-Layer Interaction (CLI) for path assignment and MAC scheduling mechanism for slot assignment to improve the mesh backbone performance for providing multimedia services to various wireless clients in a hybrid WMN. The proposed strategy measures the cross-layer parameter, such as the network capacity, using the Signal to Noise Ratio (SINR) and assigns a route that maximizes the efficiency of the multimedia services. Finally, the performance evaluation shows that the proposed cross-layer based AM-DSR protocol provides efficient QoS support for the mesh backbone enabling connections to multiple clients.

Keywords: Wireless mesh networks, mesh backbone, cross-layer issues, interference, signal strength, MAC scheduling. 


\section{Introduction}

A self-organized and self-configured Wireless Mesh Network (WMN) consists of wireless mesh routers and mesh clients that dynamically establish and maintain the wireless mesh connectivity among them. The mesh routers are powerful enough to support the power, capacity and multiple radio interfaces. The wireless mesh routers construct a stable network with minimum mobility and communicate with each other in a multi-hop fashion. The gateway/bridge function of a mesh router enables the integration of other networks, such as Internet, Wi-Fi, Wi-Max, Cellular and wireless sensor networks $[1,2]$. The hybrid mesh network supports several wireless networks on the client side and the mesh backbone maintains multimedia services to different wireless clients. Heterogeneous WMNs provide Internet access to various clients, such as Wi-Fi, Wi-MAX, cellular and ad hoc networks. The heterogeneity of the client side introduces varying transmission range, capacity and interference. Delivering Quality of Service (QoS) in routing is a complex problem in the presence of heterogeneous clients, and it needs a cross-layer solution and optimization for a hybrid network scenario [3]. The growth of the Internet usage through the mesh backbone system in a broad area causes poor signal quality due to the wireless clients with different qualities and capacities. The hybrid WMN increases the coverage due to multi-interface nodes. However, it leads to the improper utilization of the network capacities, such as dynamic network topology, varying transmission range and interference [4]. The hybrid WMN supports the QoS routing through the mesh backbone. The standard technology used to reduce the network interference and enhance the network capacity is the node deployment with multiple interfaces [5]. However, these differ in QoS requirements and suffer from inherent heterogeneity on the client side.

Recently, rapid growth has been witnessed in the mesh backbone due to the proliferation of a large number of widely available users of Internet access. The existing protocols do not consider the QoS enhancement, especially in providing access capability for various clients. Most of the existing protocols for wireless mesh networks utilize the cross-layer routing approach. It is difficult to capture the dynamics of the network capacity and establish the connection between the different layers for providing online services under a hybrid network. Furthermore, it is difficult to select efficient and stable paths for providing Internet access to various heterogeneous clients due to the dynamics of network capacity. The link scheduling delay in the MAC layer reduces the performance in the mesh backbone system.

Establishing a route to satisfy QoS from the mesh backbone to several clients is a significant research issue. This paper proposes an Adaptive MultipathDynamic Source Routing (AM-DSR) protocol that supports QoS enhancement using cross-layer approaches, such as Cross-Layer Interaction (CLI) and Medium Access Control (MAC) scheduling. The proposed AM-DSR is an extension of the DSR protocol [6] to support multipath routing and cross-layer solutions. The AM-DSR mechanism estimates the routing metrics and takes the routing decisions based on the information collected from the lower layer to provide an efficient 
platform for connecting various clients. To enable the conflict free end-to-end communication between the gateway nodes and the end users, it employs the routing information in MAC scheduling scheme for slot assignment [7]. When establishing the communication between two different networks, the link capacity matching ratio and the discovery of multiple disjoint communication routes to the gateway nodes dramatically reduces the communication latency and increases the throughput. High usage based link scheduling mechanism for contending nodes optimizes the communication delay efficiently. The paper is organized as follows. Section 2 discusses the related work. Section 3 describes the proposed AM-DSR. Section 4 shows the experimental results of the proposed AM-DSR routing and Section 5 concludes the work.

\section{Related work}

The recent advances in wireless mesh networks impose different requirements on the design of cross-layer routing. The routing techniques for mesh networks provide the optimal solution to select the gateway nodes to provide QoS guarantees to the clients $[8,9]$. A major limitation of these approaches is that it considers only the routing layer information to reduce the impact of interference on the network capacity. The main reason behind the network capacity reduction is the interference and unfairness of 802.11 MAC and moreover, it does not consider the channel conditions and the cooperative network capacity [10]. Numerous methods have been introduced to ensure the robustness; the usage of multiple radios per node improves the routing performance. An extension of Ad Hoc On-Demand distance Vector (AODV) introduced for a hybrid mesh routing that describes routing and channel allocation on network interfaces without incurring the channel interference and collision [11]. A novel cluster based QoS routing protocol supports real-time multimedia services for WMNs in [12]. Hyacinth, a multi-channel mesh routing protocol, operates multiple radios to improve the routing performance [13]. In order to balance the traffic load in the network, it applies the spanning tree routing algorithm, and includes the neighbor interface binding and interface-channel assignment. Further, it integrates the routing algorithm with the channel assignment algorithm.

A cross-layer design optimizes the performance by turning the routing and MAC layer in order to enhance the throughput of multi-hop wireless networks [14]. The efficiency of the routing mechanism can be greatly enhanced by exploiting multiple frequency channels and multiple interfaces. An alternative way to enhance the network capacity is the channel assignment and slot scheduling [15]. Identifying the limitations of the MAC layer or physical layer, and utilizing the cross-layer mechanism to overcome the limitations extend the benefits. The utilization of the network capacity can be fully achieved by faster channel switching. However, the channel switching in 802.11 MAC is not sufficient. By using multiple radios and the shortest path routing, the Multi-radio Unification Protocol (MUP) improves the performance of homogeneous nodes in wireless communication [16]. The existing protocols improve the QoS routing of the data forwarding within homogeneous 
wireless mesh clients, and limited proposals have been tried to improve the performance of the routing protocol in the presence of heterogeneous clients. Moreover, the general metrics for improving the QoS provision are not sufficient for enhancing the performance of Internet access through the mesh backbone. Thus, the proposed work takes into account the cross-layer parameters for assigning the routing path and routing information for MAC scheduling in a mesh backbone.

\section{Methodology}

The primary aim of the proposed AM-DSR protocol is to achieve QoS provisioning at both the routing and MAC layer. The cross-layer design methodology takes into account the information of multiple layers together for optimizing the performance of routing and MAC layer. When establishing both the upward and downward communication, as well as the non-neighboring layer communication, the crosslayer design method can efficiently optimize the overall network performance across the multiple layers. For example, the routing layer can report the information about the data packet both to the MAC and the transport layer. In the proposed work the cross-layer design makes inter-communication among the first three layers, such as the physical, MAC and routing layer. The parameters in the routing layer are dynamically varied according to the changes in the received signal strength and the network capacity, and moreover the slot assignment can be scheduled based on the routing information.

\subsection{System model}

The system model of WMN is shown in Fig. 1. It represents a graph G (V, C, R); V is a set of nodes that establish the network. $\mathrm{V}(\mathrm{Mg}, \mathrm{Mr}, \mathrm{Mc})$ represents the set of nodes, such as the mesh gateway nodes $(\mathrm{Mg})$, mesh routers (Mr), and mobile clients (Mc); C (c1, c2, c3) describes the connections between the nodes; c1 represents the connection between a mesh router and a gateway; c2 represents the connection between the mesh routers, and $\mathrm{c} 3$ represents the connection between the mesh router and the wireless clients.

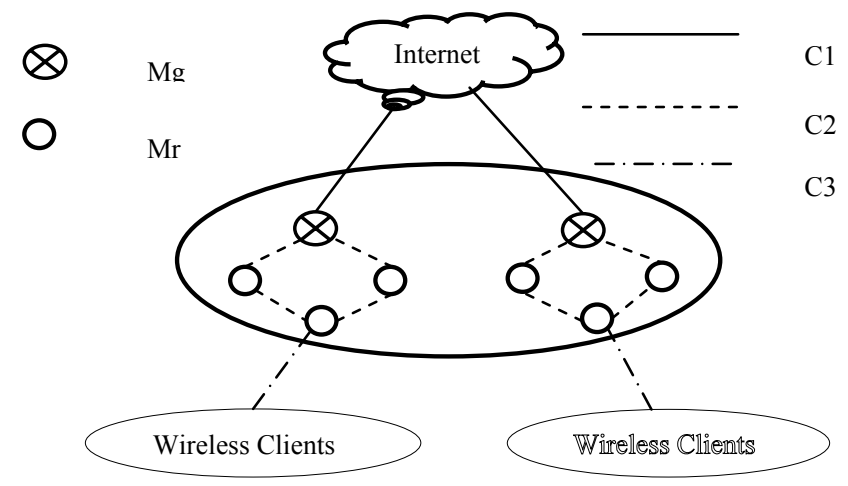

Fig. 1. Wireless mesh networks 
$R$ is the number of interfaces connected to each node $V$, and $N$ represents the number of orthogonal channels over multiple interfaces operating in 802.11. The received signal strength SINR is measured at the physical layer, and it is attached to a signal at the end of the Route REQuest (RREQ) packet. The propagated signal is converted to the frame at the MAC layer. The MAC layer obtains the signal strength of the last frame and it measures the client's capacity, which is attached to the frame and propagated to the routing layer.

\subsection{Adaptive multipath dynamic source routing protocol: AM-DSR}

The main idea of the proposed AM-DSR protocol is to support the multipath QoS routing using cross-layer mechanisms, such as CLI and link scheduling [14]. In order to determine the efficient communication routes to the gateway nodes, the CLI mechanism takes into account the cross-layer parameters, such as signal strength and capacity of the client in WMN [15]. Moreover, for reducing the communication delay, the information about the routing is employed in the link scheduling mechanism that assigns a slot to a node that accesses the medium for a long time. Thus, the proposed approach avoids the under-utilization and overutilization of the network capacity, and moreover, it avoids the unnecessary conflict among the neighbors on medium access.

\subsection{Proposed algorithms}

The proposed AM-DSR protocol supports Cross-Layer Interaction (CLI) for path assignment and MAC scheduling mechanism for slot assignment to improve the performance of the mesh networks. Figs 2 and 3 show the procedure to execute the path and the slot assignment schemes in the proposed AM-DSR protocol.

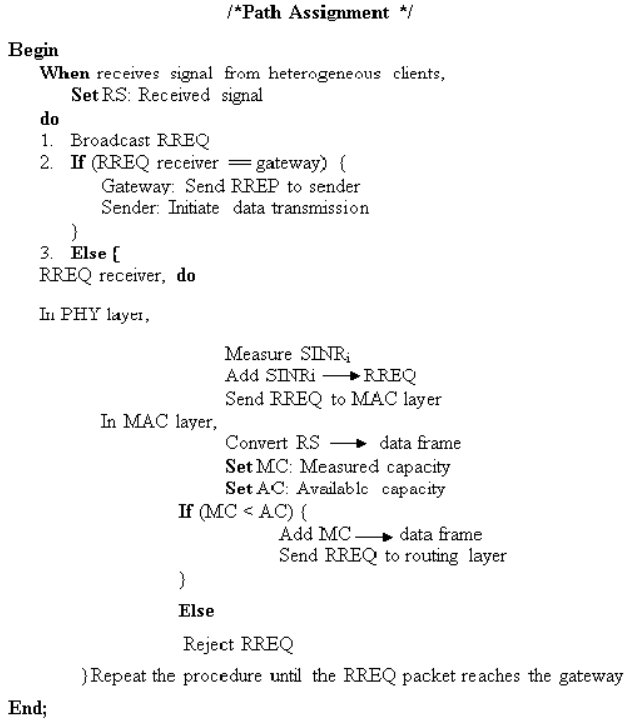

Fig. 2. Path assignment algorithm

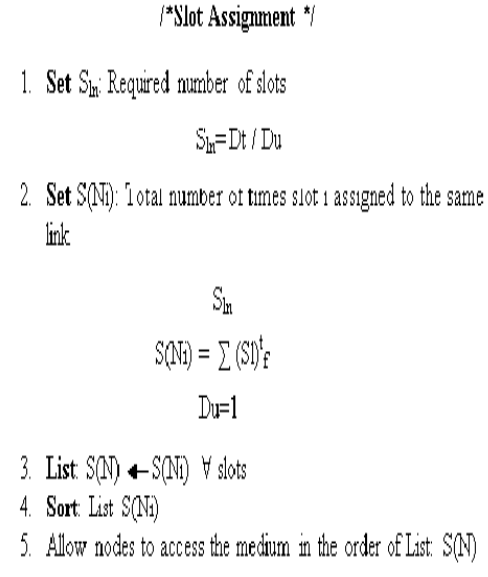

Fig. 3. Slot assignment algorithm 


\subsection{Methodology explanation}

The techniques involved in the proposed AM-DSR are path assignment, using CLI mechanism and MAC scheduling for slot assignment. The path assignment mechanism at the routing layer captures the information of the received signal strength and the network capacity correspondingly from the physical and MAC layer, while MAC scheduling mechanism for slot assignment captures the information from the routing layer.

\subsubsection{Cross-layer interaction mechanism}

Initially, the CLI mechanism measures the cross-layer parameters. The signal strength is propagated from the physical layer to the MAC layer being added as additional information. In the same way, the measured network capacity is propagated from the MAC layer to the routing layer. At the routing layer, the measured coupled network capacity is mapped with all available links to the gateway nodes during the route discovery process. Then, efficient multiple disjoint routes are selected for data transmission.

\subsubsection{Cross-layer parameter 1: Signal strength estimation}

In order to assign multiple efficient disjoint paths to the routing layer, CLI mechanism aims to investigate the available capacity of the communication routes for providing the efficient multimedia services through the gateway routers. The packet loss on capacity reduction and delay on capacity under-utilization decrease the chance of efficient transmission of the signal received from other wireless clients. It is necessary to understand the capacity of the coupled wireless client to provide the collision-free end-to-end wireless communication. For efficient wireless communication, the capacity of the coupled network is measured using the crosslayer parameter, such as the signal to noise ratio. The physical layer measures the strength of the received signal from the other wireless client and reports the signal strength information to the MAC layer [17].

The successful transmission relies on the signal-to-interference ratio at the receiver [18]. The physical layer information, i.e., SINR is propagated to the MAC layer, and it is passed as additional information with the packets. The signal to the noise ratio, SINR is defined as the ratio of the original and noise power of the received signal:

$$
\begin{aligned}
\operatorname{SINR}_{i} & =\frac{\mathrm{TP}_{i} * G_{i, j}}{\sum \mathrm{TP}_{j} * G_{j, k}+\mathrm{No}}, \\
G_{i, j} & =\frac{G_{i} G_{j}\left(S^{2}\right)}{4 \pi(\mathrm{fr})^{2} * D_{i, j}^{2}},
\end{aligned}
$$

where $\mathrm{TP}_{i}$ and $\mathrm{TP}_{j}$ represent the transmission powers of the nodes $i$ and $j$ respectively; $G_{i, j}$ and $G_{j, k}$ are the path gains of connections $\{i, j\}$ and $\{j, k\}$. No represents the noise at the receiver side. In (2) the path gain connection is derived, where $S$ represents the speed of the signal, fr represents the frequency and $D_{i, j}$ 
represents the distance between the nodes $i$ and $j$. When $D_{i, j}$ increases, the path gain decreases and it can realize the substantial decrease of SINR. Moreover, the increased power of the noise at the receiver side (No), degrades the SINR value. The noise induced during the signal transmission is due mainly to reasons, such as improper setup of the antenna with channel interference and long transmission range. Initially, all the mesh routers in the mesh backbone are highly resourceful, and the antenna is fitted with proper settings. Thus, the proposed work primarily considers the cross-layer parameter, such as the capacity of the client to take the routing decision.

\subsubsection{Cross-layer parameter 2: coupled network capacity estimation}

The signal strength is traded from the physical layer to the MAC layer and the received information from the physical layer is used to decide the capacity of the WMN client. The interference plays a vital role in the wireless mesh networks and it leads to undesirable consequences. The over-utilization of the capacity incurs data collision in the network and reduces the routing performance. The selection of the highly congested route for transmitting the low power signal received from other wireless clients reduces the signal power continuously. It is necessary to select the communication routes based on the capacity mapping concept for improving the routing performance of the mesh backbone,

$$
C=1 / \text { fr } \log \left(1+\mathrm{SINR}_{\mathrm{mb}}\right) \text {. }
$$

The long transmission of signals, using the same frequency reduces the signal strength and capacity. In (3) the SINR ${ }_{\mathrm{mb}}$ represents the received RREQ packet's signal to noise ratio within the mesh backbone. Each router in the mesh backbone measures and compares the SINR of receiving an RREQ packet with the coupled client's capacity. The high capacity communication routes are assigned for data transmission of the client WMN through the mesh backbone support.

\subsubsection{Routing techniques}

At the routing layer, three routing mechanisms, such as route discovery, route maintenance and data forwarding coupled with the link scheduling mechanism are involved. Like DSR, the AM-DSR performs the route discovery process, but it includes some modifications to improve the routing performance.

\subsubsection{Disjoint multipath discovery}

The proposed AM-DSR uses source routing, which means that each data packet contains the entire route to reach the destination. A mesh router that receives the data packet initiates the route discovery process. It broadcasts RREQ packets with additional information, such as the client's capacity measured at the MAC layer to its neighboring nodes. Each node rebroadcasts the received RREQ packet when it satisfies the following two conditions - capacity mapping and disjoint routing.

Condition 1. Capacity mapping. The link capacity factor is defined by how much the route capacity is unutilized over a particular period. It is mapped with the client WMN's capacity, which is included in the RREQ packet. If the client 
WMN's capacity is equal to or less than the route capacity, the condition is satisfied.

Condition 2. Disjoint routing. It verifies Condition 2, when a link satisfies the first condition. A node verifies whether it receives the RREQ packet with the same sequence number and rebroadcasts it already. If it broadcasts the same packet, it does not broadcast the packet that avoids the joint path routing and the channel collision due to over-utilization of the network capacity. Thus, the disjoint path discovery with the knowledge of network capacity ensures the end-to-end conflictfree communication from the mesh router to the end users.

\subsubsection{MAC scheduling}

Interference and scheduling delay are introduced when multiple nodes access the shared resource at a time. When a sender node in a path wants to communicate with the first router, it transmits the Request To Send (RTS) packet. After receiving the RTS packet, a receiving node transmits the Clear To Send (CTS) packet when the medium is in an idle state. The primary mechanism to avoid the channel interference is the delay between the slot assignments for data forwarding. If the outgoing link uses a time slot, the incoming link will not use the slot immediately. However, it increases the end-to-end delay of the entire data communication that reduces the routing performance of the large-scale networks. In order to maintain the channel quality, the proposed work schedules the link transmissions by assigning the time slots efficiently for data forwarding which avoids the channel interference [14].

Consider that each node divides the medium access time into $N$ slots. If a node accesses the medium at the $N$-th slot, no other neighbors access the medium at the same slot in the interference range. It is necessary to assign the slot perfectly in order to avoid the routing delay and the packet loss due to the interference. Find a path $(\mathrm{P})$, which is already determined by the AD-DSR protocol and contains $(R)$ number of routers. In order to assign the slots for medium access, it takes $C \in R$ routers in the path $\mathrm{P}$. Therefore, it is essential to estimate how many times a slot $\left(S_{\ln }\right)$ is assigned to achieve the total Data rate $\left(D_{\mathrm{t}}\right)$. Each node needs one slot for transmitting each unit of Data $\left(D_{\mathrm{u}}\right)$. Therefore, the total number of times a slot is assigned to this link, for transmitting the total data rate $\left(D_{t}\right)$, is given by:

$$
\begin{gathered}
S_{\ln }=D_{\mathrm{t}} / D_{\mathrm{u}}, \\
S(N)=\sum_{D_{\mathrm{u}}=1}^{S_{\ln }}(S 1)^{t} f,
\end{gathered}
$$

where $f$ represents the particular data flow. For any given slot $(t), k$ nodes have conflict over access. However, a node that uses the medium for a long time receives CTS to access the medium. Thus, only for the accessing node at a time $t, \operatorname{set}(\mathrm{Sl})^{t}=1$, otherwise it is zero. Thus, $\sum S(N)$ for all slots is equal to the total data rate of the link. 


\subsection{Link scheduling delay}

Time Division Multiple Access (TDMA) scheduling allows multiple nodes to access the same medium by dividing the frequency into different time slots. The nodes access the medium one after the other, but all the time slots cannot be allocated for the data transmission. It is because if the incoming link uses a time slot, the outgoing link will not use the slot immediately, resulting in a scheduling delay. Assume that the node A makes communication with node $\mathrm{B}$ through a router C. The link A-C is an incoming link $(\mathrm{I} / \mathrm{C})$ and $\mathrm{C}-\mathrm{B}$ is an outgoing link $(\mathrm{O} / \mathrm{G})$ for node $\mathrm{C}$. To measure the total delay induced by the router $\mathrm{C}$ on transmitting the total data rate, it is necessary to find the delay between the outgoing and incoming transmissions. If the incoming link (C-B) uses a time slot $t$, the next slot $t+1$ is not assigned to the outgoing link (A-C). In routing nodes, it is mandatory to follow the store-and-forward technique, but it is needed to optimize the MAC scheduling process for reducing the unnecessary delay in communication. In the link scheduling delay estimation, two different cases are involved.

Case 1. The slot number ( $\mathrm{Sl}) v$ is used for the outgoing link, and the slot number $u$ is used for the incoming link. The delay introduced after receiving the packet from a sender node at a relay node (r) Delay $=v-u$ if $v>u$.

In Fig. 4 the total number of slots is equal to 5. Slot 3 is used for the incoming link and slot 5 is used for outgoing links. The router $\mathrm{C}$ receives a packet from the node A at time slot $v$, but it transmits the packet to node $\mathrm{B}$ at slot $u$. When receiving the data packet, the storage time or delay induced by the router $\mathrm{C}$ is $5-3=2$.

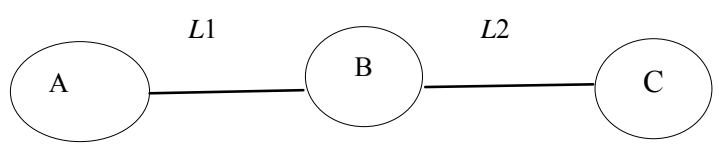

\begin{tabular}{|c|c|c|}
\hline $\mathrm{Sl}$ & $\mathrm{I} / \mathrm{C}$ & $\mathrm{O} / \mathrm{G}$ \\
\hline 1 & 0 & 0 \\
\hline 2 & 0 & 0 \\
\hline 3 & 1 & 0 \\
\hline 4 & 0 & 0 \\
\hline 5 & 0 & 1 \\
\hline
\end{tabular}

Fig. 4. Case 1: Link scheduling

Case 2. The slot number $v$ is used for the outgoing link, and the slot number $u$ is used for the incoming link. The total delay introduced at a relay node Delay $_{\mathrm{r}}=v-u+T$, if $v<u$; $T$ represents the total number of slots in a scheduling period.

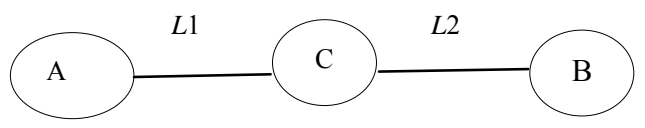

\begin{tabular}{|c|c|c|}
\hline $\mathrm{S} l$ & $\mathrm{I} / \mathrm{C}$ & $\mathrm{O} / \mathrm{G}$ \\
\hline 1 & 0 & 0 \\
\hline 2 & 0 & 0 \\
\hline 3 & 0 & 1 \\
\hline 4 & 0 & 0 \\
\hline 5 & 1 & 0 \\
\hline
\end{tabular}

Fig. 5. Case 2: Link scheduling 
Fig. 5 shows Case 2 of link scheduling, in which the total number of slots is 5 . Slot 5 is used for the incoming link and slot 3 for the outgoing link. After receiving the data packet, the storage time or the total delay induced by the router $\mathrm{C}$ is $3-5+5=3$.

\subsection{Slot assignment}

Each slot is assigned perfectly to avoid any unnecessary delay in the end-to-end data transmission. Each slot obtains different $S(N)$ values, i.e., each slot is assigned a different number of times for a particular data flow. Based on the $S(N)$ value, the slot $\mathrm{Sl}$ is in a decreasing order. Several nodes have conflicted to connect the node at time $t$, at most one of them can access slot $t$. Thus, for a node that uses a link for a long time, set $\mathrm{Sl}=1$ and for the others, set $\mathrm{Sl}=0$. Thus, this avoids unnecessary delay in link scheduling and interference.

\subsubsection{Data forwarding}

A mesh router/ gateway node replies the Route REPly (RREP) packet that is routed back to the source mesh router. A node that receives the RREP packet stores the source route in its cache for a particular time. A mesh router receives multiple RREP packets from the gateway nodes. By employing the slot assignment mechanism, it initiates the data forwarding through multiple available routes to the gateway nodes. If any link on a communication route is over-utilized, the source node is informed by a Route ERRor (RERR) packet. The node removes any route using the over-utilized link from the cache. Thus, the proposed work achieves better QoS provision for enabling connection to the client WMN. By the reduction of the medium access delay due to the slot assignment mechanism, the performance of the routing protocol is improved. Moreover, the proposed protocol is not only improving the performance of a highly stable mesh backbone system, but it is suitable for performing the on-demand routing among wireless clients. Thus, it increases the efficiency of wireless routing even under homogeneous and heterogeneous mobile networks.

\section{Performance evaluation}

The NS-2 based simulation model validates the performance of the proposed crosslayer AM-DSR protocol over a hybrid mesh network that consists of a mesh backbone and wireless clients. Thus, this work performs comparative evaluation of AM-DSR performance with the Ad Hoc On Demand Vector-Hybrid Mesh (AODV-HM) [11] in a hybrid mesh network. The AODV-HM is a similar reactive protocol proposed for hybrid mesh network that has similar abilities. The number of the traffic flow is varied with the distinct source and destination pair in the network to simulate the scenario of the simultaneous medium accessible by the nodes. Then, measure the interference and the incurred latency. Multiple scenarios are created by varying the numbers of traffic flows and nodes with fixed configuration. The 
number of disjoint paths, path length, capacity matching ratio, SINR and latency are calculated for each scenario.

\subsection{Simulation model}

The simulation model consists of 40-100 mesh routers and among them only 5-20 nodes have gateway functionality due to its high cost. These mesh routers are placed correspondingly within the $1000 \times 1000 \mathrm{~m}^{2}$ area. The ad hoc networks consist of 100 nodes within the area of $1000 \times 1000 \mathrm{~m}^{2}$, and the transmission range is $150 \mathrm{~m}$ for all nodes. The speed of each node is $25 \mathrm{~m} / \mathrm{s}$ and it pauses for $5 \mathrm{~s}$. Multimedia traffic is generated at the application layer. The network is simulated for $900 \mathrm{~s}$. The multichannel and multi-interface configuration in the network setting facilitates each node with a different number of channels and interfaces.

\subsection{Simulation results}

The simulated results of different simulation models are discussed. In order to determine the performance of the proposed AM-DSR approach, various performance metrics are evaluated. The performance evaluation results show that AM-DSR outperforms the existing protocols over wireless mesh networks.

\subsubsection{Interference impact on the communication latency}

The communication latency is defined as the number of slots taken by a node between two successful transmissions; shown in Fig. 6. It is seen that the interference and the Number of Medium Access (NMA) nodes have a direct impact on the communication latency. The ratio of signal to noise is high which means that there is minimum noise occurrence in the received signal, and the signal strength is very high. Even though the received signal strength is very high, the existing AODV-HM experiences a 13 to 30 slots delay during the data communication. However, the proposed AM-DSR experiences a delay of 2 to 18 slots only when the number of medium access nodes is high. This result shows that the proposed work reduces $40 \%$ of the delay induced by the existing AODV-HM protocol. It shows that the slot assignment algorithm minimizes the queuing delay at the MAC layer.

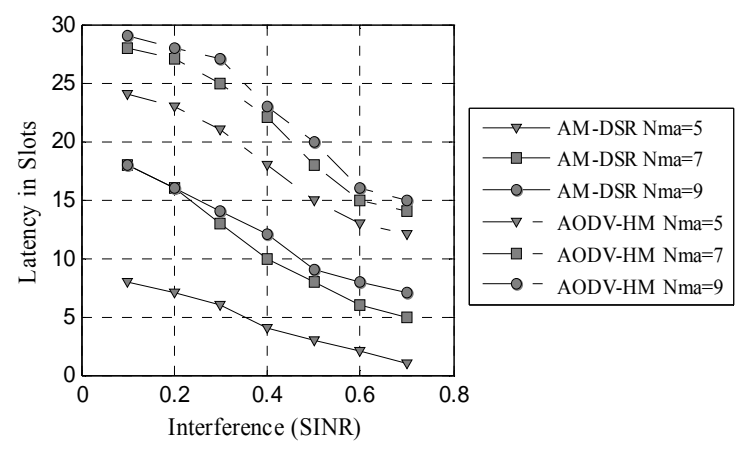

Fig. 6. Interference vs latency in slots 
4.2.2. The impact of the number of disjoint paths on the throughput

Throughput is defined as the number of bits delivered to the destination per second. The throughput is shown in Fig. 7 that represents the improved throughput when the number of disjoint paths is increased. It illustrates that the disjoint paths entirely avoid the channel interference and the delay during the slot assignment, and hence it provides efficient communication without any sudden changes in the network capacity and packet loss. The existing AODV-HM routing yields a throughput of around 20-90 kbps over the multiple disjoint routing paths due to the absence of routing information in the slot assignment. The disjoint path assignment and a single slot assignment to any link achieve aggregated throughput of around 50-140 kbps even if the Path length (Pl) is varied from 5 up to 7. This result explains that when the cross-layer information is available, the path assignment at the routing layer and the slot assignment at MAC layer improve from 30 up to $50 \mathrm{Kbps}$ under a maximum number of hop lengths.

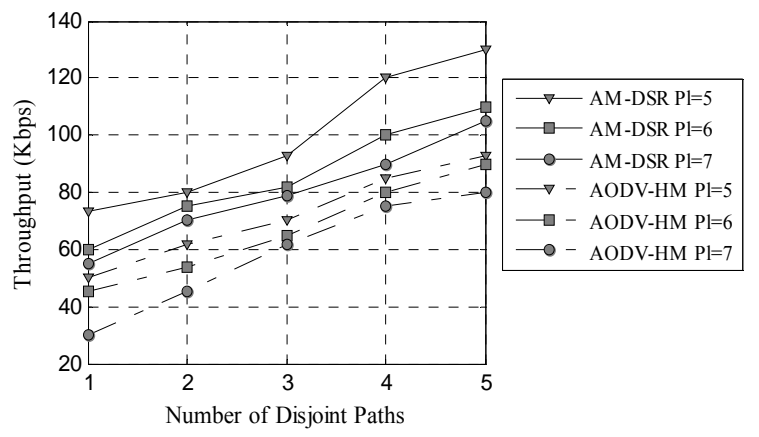

Fig. 7. Number of disjoint paths vs throughput

\subsubsection{Capacity Matching Ratio vs latency}

The Capacity Matching Ratio (CMR) is defined as the ratio between the required bandwidth of client WMN and the available bandwidth in the mesh backbone network. Fig. 8 demonstrates the impact of CMR and interference, measured in terms of SINR, on the communication latency. If the available bandwidth is less than the required bandwidth of other networks, the communication latency increases. The CMR based disjoint path assignment in AM-DSR protocol reduces the communication delay to $25-30 \%$ of the delay induced by the AODV-HM protocol. The performance of both routing protocols varies due to the consideration of CMR in a disjoint path assignment scheme. The CMR based path assignment in AM-DSR experiences a delay range from 2-0.5 $\mathrm{s}$ because it involves a single path routing. However, the AODV-HM protocol delay ranges from 3.5 up to $2 \mathrm{~s}$ under the same scenario. It shows that the consideration of the capacity matching concepts in path assignment provides opportunity to improve the routing performance significantly. 


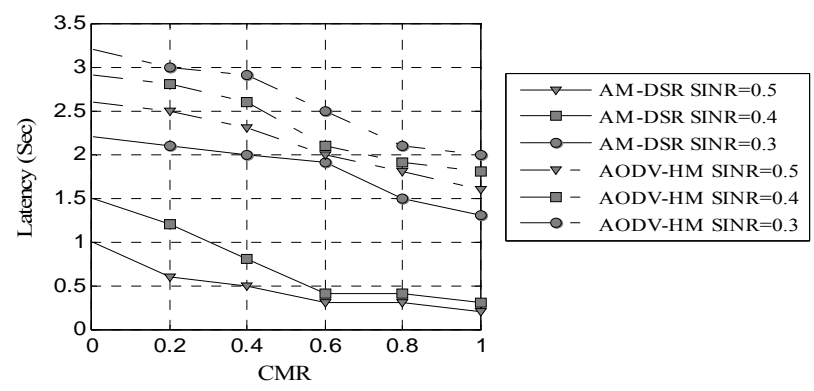

Fig. 8. CMR vs latency

4.2.4. The impact of the number of connections on the routing overhead

The routing overhead is defined as the ratio of the total number of control packets generated by the total number of data packets. The results of Fig. 9 show that the routing overhead of AM-DSR increases with the number of connections and nodes $(N)$ in the network. The routing overhead of both protocols stays close to 0.06 up to 30 connections. It is because the path includes stable mesh routers more than mesh clients that improve the network throughput in AODV-HM. The routing overhead of AM-DSR is relatively smooth when the number of connections is below 30 . With low traffic, the mesh routers have high capacity; it satisfies the requirement of the client; and the proposed AM-DSR performs similar to that of the existing AODV-HM. At the point of connections larger than 30, the routing overhead is suddenly increased, because of the saturated network. The existing AODV-HM uses the same route by adjusting the data rate. However, the AM-DSR is required to establish new routes immediately to match with the capacity of wireless clients.

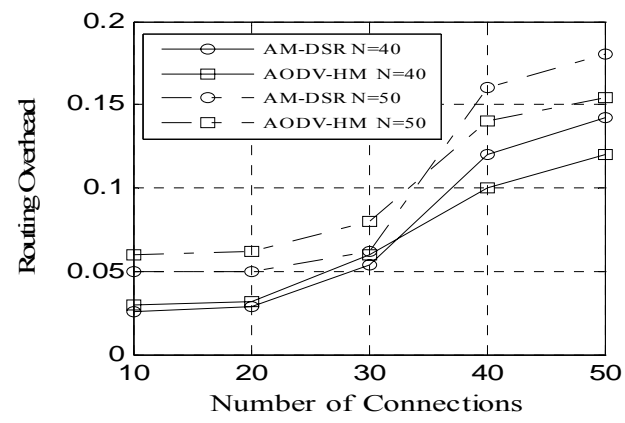

Fig. 9. Number of connections vs routing overhead

\subsubsection{SINR impact on the path optimality}

The path optimality is measured in terms of Bit Error Rate (BER). The BER refers to the percentage of incorrect bits averaged over every 100 data packets. The impact of SINR and CMR on the path optimality for 25 connections, is shown in Fig. 10. The selection of the highly congested route for transmitting the low power signal received from the other wireless clients increases BER continuously. Then it is difficult to satisfy the client's requirement with a low SINR. CMR is reduced with 
the number of NMA, and thus it increases BER in the signal that is observed in Fig. 8, between NMA 5 and 7. With an increased number of NMA, the existing AODV-HM increases the BER in the signal from 0.001 up to 0.02 ; however, the proposed AM-DSR reduces BER to $25 \%$. The slot assignment algorithm in AMDSR minimizes the queuing delay, and it employs multiple disjoint routes that avoid interference in the network. Thus it improves the CMR for each flow and reduces the BER in the signal.

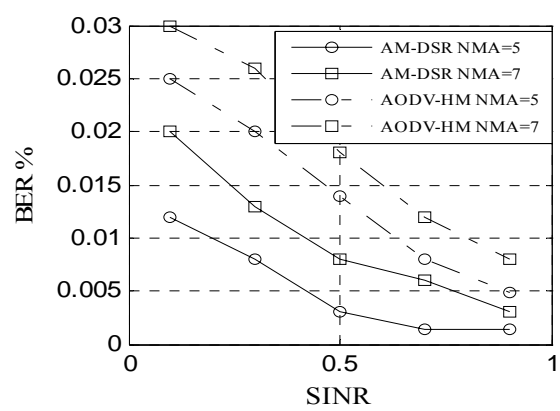

Fig. 10. SINR vs BER

4.2.6. The impact of the number of channels and radios on the network throughput

The throughput of AM-DSR increases with the number of available disjoint paths in a single radio-multi-channel network as shown in Fig. 7. The network throughput of AM-DSR and AODV-HM, varying the number of radios with 6 and 11 channels, is shown in Fig. 11. The network throughput increases with the number of interfaces because the use of multi-channels and interfaces allows more connections to transmit and receive the packets simultaneously. The slot assignment algorithm in AM-DSR works on the multi-radio network efficiently and it minimizes the queuing delay at the MAC layer by providing the priority to a node that uses a link for a long time. It causes an impact on the network throughput of AM-DSR. At the same time, the network throughput in both protocols is increased gradually, while increasing the number of channels from 6 up to 11 with five radios. Increasing the number of channels from 6 up to 11, there is no further improvement in the network throughput with two interfaces.

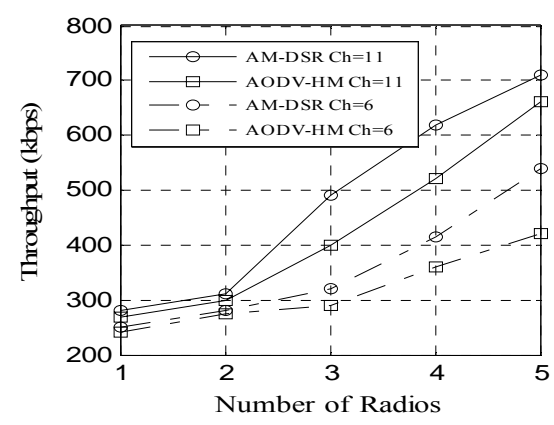

Fig. 11. Number of radios vs throughput 


\section{Comparative analysis between AM-DSR and AODV-HM}

Table 1 shows the comparative analysis of the proposed AM-DSR and the existing AODV-HM protocol.

Table 1. Comparative analysis between AM-DSR and AODV-HM

\begin{tabular}{|c|c|c|}
\hline Metrics & AM- DSR & AODV-HM \\
\hline Throughput & $\begin{array}{c}\text { Selection of high-quality multiple } \\
\text { disjoint paths improves the } \\
\text { network throughput }\end{array}$ & $\begin{array}{c}\text { Single path routing attains } \\
\text { lower throughput than AM- } \\
\text { DSR }\end{array}$ \\
\hline $\begin{array}{c}\text { MAC } \\
\text { scheduling } \\
\text { latency }\end{array}$ & $\begin{array}{c}\text { The MAC scheduling algorithm } \\
\text { provides preference to the node } \\
\text { that accesses the slot for a long } \\
\text { time }\end{array}$ & $\begin{array}{c}\text { Primary TDMA causes delay } \\
\text { in link scheduling }\end{array}$ \\
\hline Packet loss & $\begin{array}{c}\text { The selection of high capacity } \\
\text { paths incurs a small amount of } \\
\text { packet loss }\end{array}$ & $\begin{array}{c}\text { Packet loss is high due to the } \\
\text { channel interference }\end{array}$ \\
\hline $\begin{array}{c}\text { Control } \\
\text { overhead }\end{array}$ & $\begin{array}{c}\text { Rejection of duplicate packets } \\
\text { incurs less control overhead }\end{array}$ & $\begin{array}{c}\text { RREQ blind broadcasting } \\
\text { leads to high control } \\
\text { overhead }\end{array}$ \\
\hline
\end{tabular}

\section{Conclusion}

This paper proposes an AM-DSR protocol that improves the QoS, using cross-layer mechanisms, such as CLI and MAC scheduling suggested for providing efficient cooperative WMNs. The support of the cross-layer parameters in the proposed AMDSR improves the routing performance at the network layer. Moreover, it proves that it is not true that only the path length determines the communication delay, but the delay is a result of many factors, such as path length and interference. The consideration of the routing information in MAC scheduling and the selection of less interference paths avoid the unnecessary delay in communication. The proposed approach avoids the under-utilization and over-utilization of the network capacity, due to the selection of efficient disjoint communication paths, using the concept of capacity matching. Thus, the potential of the coupled wireless clients with wireless mesh backbone has increased significantly. Finally, the performance evaluation shows that the proposed AM-DSR protocol provides efficient QoS support for multiple clients.

\section{References}

1. B r u n o, R., M. C o n ti, E. Gr e g o r i. Mesh Networks: Commodity Multihop Ad Hoc Networks. - IEEE Communications Magazine, Vol. 43, March 2005, No 3, pp. 123-131.

2. Akyildiz, I., F., X. W ang, W. Wang. Wireless Mesh Networks: A Survey. - Computer Networks, Vol. 47, 2005, No 4, pp. 445-487.

3. Akyildiz, I. F., X. Wang. Cross-Layer Design in Wireless Mesh Networks. - IEEE Transactions on Vehicular Technology, Vol. 57, 2008, No 2, pp. 1061-1076. 
4. J a in, K., J. Padhye, V. P a d m a n a b h a n, L. Q i u. Impact of Interference on Multi-Hop Wireless Network Performance. - In: Proc. of 9th Annual International Conference on Mobile Computing and Networking, 2003, pp. 66-80.

5. Drave s, R., J. P a d h y e, B. Z i 1 l. Routing in Multi-Radio, Multi-Hop Wireless Mesh Networks. - In: ACM International Conference on Mobile Computing and Networking (MobiCom), 2004, pp. 114-128.

6. Broch, J., D. B. Johnso n, D. A. Maltz. The Dynamic Source Routing Protocol for Mobile Ad Hoc Networks, June 1999 http://www.ietf.org /internet drafts/draft-ietf-manet-dsr-03.txt

7. Cheng, M., Q. Ye, L. Ca i. Cross-Layer Schemes for Reducing Delay in Multihop Wireless Networks. - IEEE Transactions on Wireless Communications, Vol. 12, 2013, No 2, pp. 928-937.

8. A oun, B., R. B outaba, Y. Iraqi, G. Kenward. Gateway Placement Optimization in Wireless Mesh Networks with QoS Constraints. - IEEE Journal on Selected Areas in Communications, Vol. 24, 2006, No 11, pp. 2127-2136.

9. Z h o u, P., X. W a n g, B. S. M a n o j, R. R a o. On Optimizing Gateway Placement for Throughput in Wireless Mesh Networks. - EURASIP Journal on Wireless Communications and Networking, 2010, doi:10.1155/2010/368423.

10. Ath a nasiou, G., T. Korakis, O. Ercetin and, L. T a s s i u las. Dynamic Cross-Layer Association in 802.11-Based Mesh Networks. - In: Proc. of 26th IEEE International Conference on Computer Communications, 2007, pp. 2090-2098.

11. P i r z a d a, A. A., M. P ortm a n n, J. In d u 1 s k a. Hybrid Mesh Ad Hoc On-Demand Distance Vector Routing Protocol. - In: Proc. of 13th Australasian Computer Science Conference, 2007.

12. Bemmoussat, Chems-eddine, Fedoua Didi, Mohamed Feham. Efficient Routing Protocol to Support QoS in Wireless Mesh Networks. - International Journal of Wireless \& Mobile Networks (IJWMN), Vol. 4, 2012, No 5, pp. 89-104.

13. R a n iw a la, A., T. C. Ch i u e h. Architecture and Algorithms for an IEEE 802.11-based MultiChannel Wireless Mesh Network. - In: Proc. of 24th Annual Joint Conference of the IEEE Computer and Communications Societies (INFOCOM'05), Vol. 3, 2005, pp. 2223-2234.

14. Uddin, M. Forkan, C. Rose $\mathrm{nberg}$, W. Zhuan. Joint Configuration of Routing and Medium Access Parameters in Wireless Networks. GLOBECOM, 2009, pp.1-8.

15. Y u, H., P. Moh a patra, X. Li u. Channel Assignment and Link Scheduling in Multi-Radio Multi-Channel Wireless Mesh Networks. - Mobile Networks and Applications, Vol. 13, 2008, No 1-2, pp. 169-185.

16. A d y a, A., P. B a h 1, J. P a d h y e, A. W o $1 \mathrm{~m}$ a n, L. Z h o u. A Multi-Radio Unification Protocol for IEEE 802.11 Wireless Networks. - In: Proc. of 1st International Conference on Broadband Networks, 2004, pp. 344-354.

17. S h a b d a n o v, S., P. M itra n, C. R o s e n b e r g. Cross-Layer Optimization Using Advanced Physical Layer Techniques in Wireless Mesh Networks. - IEEE Transactions on Wireless Communications, Vol. 11, 2012, No 4, pp.1622-1631. 